\title{
LOCAL UNCERTAINTY INEQUALITIES FOR COMPACT GROUPS
}

\author{
JOHN F. PRICE AND ALLADI SITARAM
}

(Communicated by Richard R. Goldberg)

Abstract. Conditions are established on $\alpha, \beta \in \mathbf{R}$ for there to exist a constant $K=K(\alpha, \beta)$ such that

$$
\sum_{\gamma \in E} d(\gamma) \operatorname{tr}\left(\hat{f}(\gamma)^{*} \hat{f}(\gamma)\right) \leq K\left(\sum_{\gamma \in E} d(\gamma)^{2}\right)^{\alpha}\left\|w^{\beta} f\right\|_{2}
$$

for all $f \in L^{1}(G)$ and $E \subseteq \hat{G}$ where $G$ is a compact metric group, $\hat{G}$ is its dual, $\hat{f}$ is the Fourier transform of $f$ and $w: G \rightarrow \mathbf{R}^{+}$is the function taking $x \in G$ to the area of the ball in $G$ with centre $e$ and $x$ on its boundary. This is followed by a partial analogy for compact riemannian manifolds.

1. Introduction. The following is a special case of a result in [6] for multiple Fourier series: given $\alpha, \beta \in \mathbf{R}$ and $k \in \mathbf{Z}^{+}=\{1,2, \ldots\}$, there exists a constant $K$ such that

$$
\left(\sum_{n \in E}|\hat{f}(n)|^{2}\right)^{1 / 2} \leq K|E|^{\alpha}\left|\left\||x|^{k \beta} f\right\|_{2}\right.
$$

for all $f \in L^{1}\left(\mathrm{~T}^{k}\right)$ and all finite $E \subseteq \mathbf{Z}^{k}$ if and only if $\alpha, \beta$ satisfy

$$
\beta<1 / 2, \quad \alpha \geq 0 \text { and } \alpha \geq \beta \text {. }
$$

$\left(|E|\right.$ denotes the cardinality of $E$ and the function $|x|$ is defined on $\mathbf{T}^{k}$ by identifying this group with $\left(-\frac{1}{2}, \frac{1}{2}\right]^{k}$.) This is a local uncertainty inequality in the sense that concentration of $f$ limits the localization of $\hat{f}$ on any given set. The main result below, Theorem 2.4, is a direct analogue valid for all compact metrizable groups. We then give a somewhat less complete version for compact analytic manifolds. Local uncertainty inequalities for certain noncompact Lie groups are given in [7] and for $\mathbf{R}^{d}$ in $[\mathbf{1}, \mathbf{4}, \mathbf{5}]$.

2. Compact metric groups. Throughout this section $G$ will be a compact nonfinite metric group equipped with normalized Haar measure $d \mu$ and $\hat{G}$ will be its unitary dual, that is, $\hat{G}$ is a maximal set of pairwise inequivalent unitary irreducible continuous representations of $G$. Denote by $\nvdash_{\gamma}$ the (finite-dimensional) Hilbert space on which $\gamma \in \hat{G}$ acts. As usual, the Fourier series of $f \in L^{\mathbf{1}}(G)$ is written as

$$
f \sim \sum_{\gamma \in \hat{G}} d(\gamma) \operatorname{tr}(\hat{f}(\gamma) \gamma(\cdot))
$$

where $d(\gamma)$ is the dimension of $\nvdash_{\gamma}$ and $\hat{f}(\gamma)=\int_{G} f(x) \gamma\left(x^{-1}\right) d \mu(x)$. Our first concern is to introduce a function which plays the role of $|x|$ when $G=\mathrm{T}^{k}$.

Received by the editors March 16, 1987.

1980 Mathematics Subject Classification (1985 Revision). Primary 43A15. 
Let $d(\cdot, \cdot)$ be a metric on $G$ which describes its topology. Without loss of generality assume that the metric is normalized so that $\sup \{d(e, x): x \in G\}=1$. Since $G$ is compact, this supremum is actually attained. Denote $[0,1]$ by $I$ and define a nondecreasing measurable function $A: I \rightarrow I$ by

$$
A(r)=\mu\left(B_{r}\right) \text { where } B_{r}=\{x \in G: d(x, e) \leq r\} .
$$

Since $G$ is nonfinite and compact, $A(0)=0$. Also the fact that if $r_{n} \searrow r$ as $n \rightarrow \infty$ for some sequence $\left(r_{n}\right)$, then $B_{r}=\bigcap_{n} B_{r_{n}}$, shows that $A$ is continuous on the right.

Define a continuous map $\phi: G \rightarrow I$ by $\phi(x)=d(x, e)$. Also let $B_{r}^{\prime}$ denote the complement of $B_{r}$ in $G$.

2.1 LemMA. For any $\varepsilon>0, \mu\{x \in G: A(\phi(x))<\varepsilon\} \leq \varepsilon$.

REMARK. $A$ is right continuous, $\phi$ is continuous and so $A \circ \phi$ is measurable. Hence the set in Lemma 2.1 is measurable.

ProOF OF 2.1. Given $\varepsilon>0$, let $Y=\{x \in G: A(\phi(x))<\varepsilon\}$. Since always $y \in$ $B_{\phi(y)}, Y \subseteq \bigcup\left\{B_{\phi(x)}: x \in Y\right\}$. On the other hand, suppose $y \in \bigcup\left\{B_{\phi(x)}: x \in Y\right\}$, that is, $y \in B_{\phi(x)}$ for some $x \in Y$. Hence $\phi(y) \leq \phi(x)$ and so $A(\phi(y)) \leq A(\phi(x))<$ $\varepsilon$, with the conclusion that $y \in Y$. This has established the fact that

$$
Y=\bigcup\left\{B_{\phi(x)}: x \in Y\right\},
$$

from which the conclusion in Lemma 2.1 is a straightforward consequence.

2.2 LemMA. The function $w=A \circ \phi: G \rightarrow I$ is measurable and satisfies

$$
\int_{B_{r}} w^{-\theta} d \mu \leq \frac{A(r)^{1-\theta}}{1-\theta} \text { for } 0 \leq \theta<1
$$

and

for each $r>0$.

$$
\left\|w^{-\theta} 1_{B_{r}^{\prime}}\right\|_{\infty} \leq A(r)^{-\theta} \text { for } \theta \geq 0
$$

Further, for $\theta \leq 0, w^{-\theta}$ is continuous and hence bounded since $G$ is compact. Consequently

$$
\int_{G} w^{-\theta} d \mu<\infty \text { for } \theta \leq 0
$$

PrOOF. We just give a sketch of the proof of the first inequality. Define $G_{t}=$ $\left\{x \in G: w(x)^{-1}>t\right\}$ for $t \geq 0$. By the change of variable formula [3, (21.72)],

$$
\int_{B_{r}} w^{-\theta} d \mu=\int_{0}^{\infty} \theta t^{\theta-1} \mu\left(G_{t} \cap B_{r}\right) d t .
$$

First consider the integral $I_{1}=\int_{0}^{1 / A(r)} \theta t^{\theta-1} \mu\left(G_{t} \cap B_{r}\right) d t$. Since $\mu\left(G_{t} \cap B_{r}\right) \leq$ $\mu\left(B_{r}\right)=A(r)$, we have

$$
I_{1} \leq A(r) \int_{0}^{1 / A(r)} \theta t^{\theta-1} d t=A(r)^{1-\theta} .
$$

Now consider $I_{2}=\int_{1 / A(r)}^{\infty} \theta t^{\theta-1} \mu\left(G_{t} \cap B_{r}\right) d t$. Whenever $t>1 / A(r), G_{t} \subseteq B_{r}$. (Let $x \in G_{t}$; then $A(\phi(x))<t^{-1}<A(r)$ and so $\phi(x) \leq r$, that is, $x \in B_{r}$.) Hence

$$
I_{2}=\int_{1 / A(r)}^{\infty} \theta t^{\theta-1} \mu\left(G_{t}\right) d t .
$$


Lemma 2.1 shows that $\mu\left(G_{t}\right) \leq 1 / t$ and so

$$
I_{2} \leq \int_{1 / A(r)}^{\infty} \theta t^{\theta-1} t^{-1} d t=\frac{A(r)^{1-\theta} \theta}{1-\theta} .
$$

Combining (2.2) and (2.3) and substituting in (2.1) gives the required inequality.

REMARK. In the above, notice that $A(r)>0$ for $r>0$. This is because $B_{r}$ is a neighbourhood of $e$ for each $r>0$ and so has positive Haar measure.

2.3 AsSUMPTION. To obtain a more complete analogy with the result (1.1) for $\mathbf{T}^{k}$ we will need $G$ and its metric to satisfy the following: there exists $\lambda>0$ such that for all $s \in[0,1]$ there exists $r \in[0,1]$ with

$$
s \leq A(r) \leq \lambda s
$$

A wide class of groups, including the connected compact Lie groups, can be equipped with compatible metrics so that this condition is satisfied.

Whenever $E \subseteq \hat{G}$, define $|E|_{2}=\left(\sum_{\gamma \in E} d(\gamma)^{2}\right)^{1 / 2}$.

2.4 THEOREM. Let $G$ be a compact metric group and suppose $\alpha, \beta \in \mathbf{R}$. Consider the following inequality: there exists a constant $K=K(\alpha, \beta)$ such that

$$
\left(\sum_{\gamma \in E} d(\gamma) \operatorname{tr}\left(\hat{f}(\gamma) \hat{f}(\gamma)^{*}\right)\right)^{1 / 2} \leq K|E|_{2}^{2 \alpha}\left\|w^{\beta} f\right\|_{2}
$$

for all finite $E \subseteq \hat{G}$ and all $f \in L^{1}(G)$.

(i) The inequality is valid for $\{(\alpha, \beta): \alpha \geq 0, \beta<1 / 2$ and $\beta<\alpha\}$ and $\{(\alpha, \beta): \alpha=0, \beta \leq 0\}$.

(ii) If $G$ also satisfies Assumption 2.3 the inequality continues to hold when $0<\alpha=\beta<1 / 2$.

2.5 REMARK. When $G=\mathbf{T}^{k}$ the function $w=A \circ \phi$ can be chosen to equal $|x|^{k}$. Furthermore, in this case $|E|_{2}$ reduces to $|E|$.

PROOF (OF THEOREM 2.4). We first introduce spaces which are nonabelian analogues of $l^{p}(\mathbf{Z})$. Full details are available in Hewitt and Ross [2]. Let $\mathbb{E}$ be the set of functions $\psi$ on $\hat{G}$ with $\psi(\gamma) \in B\left(\mathcal{H}_{\gamma}\right)$ for $\gamma \in \hat{G}$, where $B\left(\mathcal{H}_{\gamma}\right)$ is the space of bounded linear operators on $\mathfrak{H}_{\gamma}$. For $1 \leq p \leq \infty$, let $\mathfrak{E}_{p}$ be the normed subspace of $\mathfrak{E}$ as in $[2,(28.24)]$ : denote the corresponding norm by $\|\cdot\|_{p}$. In particular, $\|\psi\|_{2}=\left(\sum_{\gamma \in \hat{G}} d(\gamma) \operatorname{tr}\left(\psi(\gamma)^{*} \psi(\gamma)\right)\right)^{1 / 2}$ and $\|\psi\|_{\infty}=\sup _{\gamma \in \hat{G}}\|\psi(\gamma)\|$, where $\|\psi(\gamma)\|$ is the operator norm of $\psi(\gamma)$. Let $E$ be a finite subset of $\hat{G}$.

(i) Throughout the proof of part (i) we assume that $\alpha, \beta \in \mathbf{R}$ satisfy $\alpha \geq 0$ and $\beta<1 / 2$. Define $\psi_{E} \in \mathbb{E}$ by $\psi_{E}(\gamma)=I_{d(\gamma)}$, the identity operator in $B\left(\not H_{\gamma}\right)$, when $\gamma \in E$ and 0 otherwise. For $p \in[1, \infty]$ define $p^{\prime}$ and $p^{\#}$ by $p^{\prime}=p(p-1)^{-1}$ and $p^{\#}=2 p(p-2)^{-1}$. 
Given $f \in L^{1}$, the following sequence of inequalities follows from (28.33) and (31.22) of [2] and Hölder's inequality:

$$
\begin{aligned}
\left(\sum_{\gamma \in E} d(\gamma) \operatorname{tr}\left(\hat{f}(\gamma) \hat{f}(\gamma)^{*}\right)\right)^{1 / 2} & =\left\|\psi_{E} \hat{f}\right\|_{2} \\
& \leq\left\|\psi_{E}\right\|_{p^{\#}}\|\hat{f}\|_{p} \quad(\text { where } 2 \leq p \leq \infty) \\
& \leq\left\|\psi_{E}\right\|_{p^{\#}}\|f\|_{p^{\prime}} \\
& \leq\left(\sum_{\gamma \in E} d(\gamma)^{2}\right)^{1 / p^{\#}}\left\|(A \circ \phi)^{-\beta}\right\|_{p^{\#}}\left\|(A \circ \phi)^{\beta} f\right\|_{2} .
\end{aligned}
$$

Let $\alpha=1 / p^{\#}$. By Lemma 2.2, $\left\|(A \circ \phi)^{-\beta}\right\|_{p^{\#}}$ is finite when $\beta p^{\#}<1$ (that is, when $\beta<\alpha)$ and $\alpha=1 / p^{\#}>0$, and when $\beta \leq 0$ and $\alpha=0$.

In the preceding argument we required $2 \leq p \leq \infty$ which implies $0 \leq \alpha \leq 1 / 2$. Hence $\beta<1 / 2$ is also required. Thus for the pairs $\{(\alpha, \beta): 0 \leq \alpha \leq 1 / 2, \beta<$ $1 / 2, \beta<\alpha\}$ and $\{(0, \beta): \beta \leq 0\}$ we have the required inequality with the constant $K=\left\|(A \circ \phi)^{-\beta}\right\|_{1 / \alpha}$. Since $|E|_{2} \geq 1$ for nonempty $E,|E|_{2}^{2 \alpha} \leq|E|_{2}^{2 \alpha^{\prime}}$ whenever $\alpha \leq \alpha^{\prime}$. This completes part (i) because the validity of the inequality (2.5) for a pair $(\alpha, \beta)$ implies its validity for all pairs $\left(\alpha^{\prime}, \beta\right)$, with $\alpha^{\prime} \geq \alpha$, with the same constant.

(ii) Up until the last step, the proof of part (ii) follows that of Theorem $1^{\prime}$ of [4] or Theorem 1.1 of [7]. We then invoke (2.4). Given $r \in(0,1)$, let $f_{1}=f 1_{B_{r}}$ and $f_{2}=f-f_{1}$. Then

$$
\left(\sum_{\gamma \in E} d(\gamma) \operatorname{tr}\left(\hat{f}(\gamma)^{*} \hat{f}(\gamma)\right)\right)^{1 / 2} \leq I_{1}+I_{2}
$$

where

$$
\begin{aligned}
I_{1} & =\left(\sum_{\gamma \in E} d(\gamma) \operatorname{tr}\left(\hat{f}_{1}(\gamma)^{*} \hat{f}_{1}(\gamma)\right)\right)^{1 / 2} \\
& \leq|E|_{2}\left\|\hat{f}_{1}\right\|_{\infty} \leq|E|_{2}\left\|f_{1}\right\|_{1} \leq|E|_{2}\left\|w^{-\beta}\right\|\left\|_{2}\right\| w^{\beta} f_{1} \|_{2} \\
& \leq|E|_{2} \frac{A(r)^{-\beta+1 / 2}}{(1-2 \beta)^{1 / 2}}\left\|w^{\beta} f_{1}\right\|_{2}
\end{aligned}
$$

and

$$
\begin{aligned}
I_{2} & =\left(\sum_{\gamma \in E} d(\gamma) \operatorname{tr}\left(\hat{f}_{2}(\gamma)^{*} f_{2}(\gamma)\right)\right)^{1 / 2} \\
& \leq\left\|f_{2}\right\|_{2} \leq\left\|w^{-\beta} 1_{B_{r}^{\prime}}\right\|_{\infty}\left\|w^{\beta} f_{2}\right\|_{2} \leq A(r)^{-\beta}\left\|w^{\beta} f_{2}\right\|_{2} .
\end{aligned}
$$

(In both cases the final inequality follows from Lemma 2.2.) Hence

$$
\left(\sum_{\gamma \in E} d(\gamma) \operatorname{tr}\left(\hat{f}(\gamma)^{*} \hat{f}(\gamma)\right)\right)^{1 / 2} \leq A(r)^{-\beta}\left(\frac{A(r)^{1 / 2}|E|_{2}}{(1-2 \beta)^{1 / 2}}+1\right)\left\|w^{\beta} f\right\|_{2}
$$


using the fact that $\left\|w^{\beta} f_{1}\right\|_{2},\left\|w^{\beta} f_{2}\right\|_{2} \leq\left\|w^{\beta} f\right\|_{2}$.

The proof is completed by applying inequality (2.4) (that is, Assumption 2.3) with $s=\left|E_{2}\right|^{-2}$. If $E$ is nonempty (which we can of course assume), then $|E|_{2} \geq 1$ and so $s=\left|E_{2}\right|^{-2} \leq 1$. Thus by inequality (2.4), there exists $r$ such that $A(r) \leq$ $\lambda|E|_{2}^{-2}$ and $A(r) \geq|E|_{2}^{-2}$. Thus $A(r)^{1 / 2} \leq \lambda^{1 / 2}|E|_{2}^{-1}$ and $A(r)^{-\beta} \leq|E|_{2}^{2 \beta}$ which, upon substitution into (2.6), gives

$$
\left(\sum_{\gamma \in E} d(\gamma) \operatorname{tr}\left(\hat{f}(\gamma) \hat{f}(\gamma)^{*}\right)\right)^{1 / 2} \leq|E|_{2}^{2 \beta}\left(\frac{\lambda^{1 / 2}}{(1-2 \beta)^{1 / 2}}+1\right)\left\|w^{\beta} f\right\|_{2}
$$

as required.

3. Compact manifolds. In this section $X$ will denote a compact oriented riemannian manifold. A suitable reference is Warner [10]. Let $d$ denote the metric on $X$ induced by the given riemannian structure on $X$. Fix $x_{0} \in X$ and write $\phi(x)=d\left(x, x_{0}\right)$ for $x \in X$.

Denote the Laplace-Beltrami operator (with respect to the given riemannian structure) on $C^{\infty}(X)$, the space of infinitely differentiable functions on $X$, by $\Delta$. The spectrum $\Lambda$ of $\Delta$ is of the form $\Lambda=\left\{\lambda_{1}, \lambda_{2}, \ldots\right\}$, where $0 \leq \lambda_{1}<\lambda_{2}<\cdots$. Let $\mathscr{H}_{\lambda}$ be the eigenspace corresponding to $\lambda \in \Lambda$. Then $d(\lambda)=\operatorname{dim} \mathscr{H}_{\lambda}<\infty$ and

$$
L^{2}(X)=\bigoplus \sum_{\lambda \in \Lambda} \nVdash_{\lambda}
$$

Fix an orthonormal basis $\phi_{\lambda}^{(1)}, \ldots, \phi_{\lambda}^{d(\lambda)}$ for each $\not_{\lambda}$ and define $c(\lambda)$ by

$$
c(\lambda)=\max \left\{\left\|\phi_{\lambda}^{(j)}\right\|_{\infty}: j \in\{1, \ldots, d(\lambda)\}\right\} .
$$

(For simplicity we suppress the fact that $c(\lambda)$ depends upon the chosen basis.)

For each subset $E \subseteq \Lambda$ denote the orthogonal projection of $L^{2}(X)$ onto $\bigoplus \sum_{\lambda \in E} \nvdash_{\lambda}$ by $P_{E}$. (When $E$ is a singleton $\{\lambda\}$, denote $P_{E}$ by $P_{\lambda}$.) Suppose $0 \leq \theta<1 / 2$ and define $K_{\theta}$ by

$$
K_{\theta}=\left\|(A \circ \phi)^{-\theta}\right\|_{2},
$$

where $A(r)$ is the volume (in the canonical riemannian measure induced by the riemannian structure) of the set $\left\{x \in X: d\left(x, x_{0}\right) \leq r\right\}$. As in Lemma $2.2, K_{\theta}<\infty$ since $0 \leq \theta<1 / 2$.

Let $f \in L^{2}(X)$. Then

$$
\begin{aligned}
\left\|P_{\lambda} f\right\|_{2}^{2} & =\sum_{j=1}^{d(\lambda)}\left(\int_{X} \overline{f \phi_{\lambda}^{(j)}}\right)^{2} \\
& \leq c(\lambda)^{2} d(\lambda)\left(\int_{X}|f|\right)^{2} \leq K_{\theta}^{2} c(\lambda)^{2} d(\lambda)\left\|(A \circ \phi)^{\theta} f\right\|_{2}^{2} .
\end{aligned}
$$

Hence, whenever $E \subseteq \Lambda$,

$$
\begin{aligned}
\left\|P_{E} f\right\|_{2}^{2} & \leq K_{\theta}^{2} \sum_{\lambda \in E} c(\lambda)^{2} d(\lambda)\left\|(A \circ \phi)^{\theta} f\right\|_{2}^{2} \\
& =K_{\theta}^{2} \mu(E)^{2}\left\|(A \circ \phi)^{\theta} f\right\|_{2}^{2}
\end{aligned}
$$

where $\mu(E)=\left(\sum_{\lambda \in E} d(\lambda) c(\lambda)^{2}\right)^{1 / 2}$. 
In summary, with notation as above,

$$
\left\|P_{E} f\right\|_{2} \leq K_{\theta} \mu(E)\left\|(A \circ \phi)^{\theta} f\right\|_{2}
$$

for $f \in L^{2}(X), E \subseteq \Lambda$ and $0 \leq \theta<1 / 2$ where $K_{\theta}<\infty$, a local uncertainty inequality directly analogous to (1.1) and Theorem 2.4 .

3.1 The TWO-DIMENSIONAL SPHERE. Suppose $X=S^{2}$, the two-dimensional sphere, with the usual riemannian structure. In spherical coordinates

$$
S^{2}=\{(\alpha, \beta): 0 \leq \alpha<2 \pi, 0 \leq \beta \leq \pi\}
$$

with the usual identifications. The eigenvalues of the Laplace-Beltrami operator are $n(n+1)$ with $n \in \mathbf{N}=\{0\} \cup \mathbf{Z}^{+}$and the corresponding eigenspaces $\mathcal{H}_{n(n+1)}$ have dimension $2 n+1[8]$. Let $\left\{Y_{n}^{m}:-n \leq m \leq n, m \in \mathbf{Z}\right\}$ be the associated spherical functions: they form a column of entry functions for the usual description of the $(2 n+1)$-dimensional representation of $S U(2)[9]$ and thus satisfy $\left\|Y_{n}^{m}\right\|_{\infty} \leq 1$.

The functions $\left\{(2 n+1)^{1 / 2} Y_{n}^{m}:-n \leq m \leq n, m \in \mathbf{Z}\right\}$ make up an orthonormal basis for $H_{n(n+1)}$ and so, with respect to this basis, $c_{n}=c(n(n+1)) \leq(2 n+1)^{1 / 2}$. The metric $d(\cdot, \cdot)$ with respect to the usual riemannian structure on $S^{2}$ is just the euclidean distance along great circles. Define $\phi$ on $S^{2}$ by $\phi(\alpha, \beta)=\beta$, that is, the geodesic distance between the pole $(0,0)$ and $(\alpha, \beta)$. Then

$$
(A \circ \phi)(\alpha, \beta)=2 \pi(1-\cos \beta),
$$

the surface area of the cap $\left\{\left(\alpha, \beta^{\prime}\right): 0 \leq \alpha<2 \pi, 0 \leq \beta^{\prime} \leq \beta\right\}$. Suppose $E \subseteq N$; with the above notation, (3.1) becomes

$$
\left\|P_{E} f\right\|_{2} \leq K_{\theta}\left(\sum_{n \in E}(2 n+1)^{2}\right)^{1 / 2} \int_{S^{2}}(2 \pi(1-\cos \beta))^{2 \beta}|f(\alpha, \beta)|^{2} d \mu
$$

for $f \in L^{2}\left(S^{2}\right)$ and $0 \leq \theta<1 / 2$ where

$$
\begin{aligned}
K & =\left(\int_{S^{2}}|A \circ \phi|^{-2 \theta}\right)^{1 / 2} \\
& =\int_{0}^{2 \pi} \int_{0}^{\pi}(2 \pi(1-\cos \beta))^{-2 \theta} \sin \beta d \beta d \alpha \\
& =2(2 \pi)^{-2 \theta}(1-2 \theta)^{-1}
\end{aligned}
$$

and $d \mu$ is the (riemannian) measure given by $d \mu=\sin \beta d \beta d \alpha$.

ACKNOWLEDGEMENT. The second-named author thanks the University of New South Wales for its hospitality during the preparation of this paper. (The visit was supported by the Australian Research Grant Scheme.) He is also thankful to T. Ramakrishna Rao for useful conversations.

\section{REFERENCES}

1. W. G. Faris, Inequalities and uncertainty principles, J. Math. Phys. 19 (1978), 461-466.

2. E. Hewitt and K. A. Ross, Abstract harmonic analysis. vol. II, Springer-Verlag, Berlin, 1970.

3. E. Hewitt and K. Stromberg, Real and abstract analysis, Springer-Verlag, New York, 1965.

4. J. F. Price, Inequalities and local uncertainty principles, J. Math. Phys. 27 (1983), 1711-1714.

5. __ Sharp local uncertainty inequalities, Studia Math. 85 (1986), 37-45. 
6. J. F. Price and P. C. Racki, Local uncertainty inequalities for Fourier series, Proc. Amer. Math. Soc. 93 (1985), 245-251.

7. J. F. Price and A. Sitaram, Local uncertainty inequalities for locally compact groups, Trans. Amer. Math. Soc. (to appear).

8. A. Terras, Harmonic analysis on symmetric spaces and applications, vol. I, Springer-Verlag, New York, 1985.

9. N. J. Vilenkin, Special functions and the theory of group representations, Transl. Math. Monographs, vol. 22, Amer. Math. Soc., Providence, R.I., 1968.

10. F. W. Warner, Foundations of differentiable manifolds and Lie groups, Scott-Foresmann, 1971.

SChOOL OF MAThEMATICS, UNIVERSity OF NEW SOUTh WALES, Kensington, N.S.W. 2033 Australia

STAT-MATH UNit, INDiAN STATISTICAL INSTITUTE, BANGaLORE 560059, INDia 